\title{
Early triaging using the Modified Early Warning Score (MEWS) and dedicated emergency teams leads to improved clinical outcomes in acute emergencies
}

\author{
Authors: Amar Patel, Saad Hassan, Abid Ullah, Tahir Hamid and Hannah Kirk
}

\section{Aims}

The Modified Early Warning Score (MEWS) is a well-established score to guide the early involvement of medical on-call doctors in managing unwell patients in acute emergencies. The MEWS score is based on data derived from four physiological readings including systolic blood pressure, heart rate, respiratory rate, body temperature and level of consciousness.

\section{Methods}

At our institute, the MEWS emergency team (MET) was introduced in 2008 along with a dedicated cardiac arrest team. The MET responds within 15 minutes once alerted by the nursing staff about a patient's MEWS score of seven or above. The team consists of the on-call medical registrar, on-call HDU doctor and critical outreach team. Data were collected from hospital medical records about cardiac arrest and MET calls, over a period of 12 months between March 2013 and February 2014.

\section{Results}

On the introduction of the MET in 2008, 257 cardiac arrest calls and 30 MET calls were made. Conversely, between March 2013 and February 2014 the number of MET calls had increased to 932, whilst cardiac arrest calls had significantly reduced to 119 $(\mathrm{P}=<0.0001)$.

A total of 932 MET calls were activated for 705 patients over a period of 12 months. All calls were attended within 15 minutes. The median age of patients was 76 (64-83) years. 820 calls $(88 \%)$ resulted in the optimisation of treatment, with increased frequency of observations and adjustment of therapy. 68 patients $(9.6 \%)$ were transferred to level 2 care (high-dependency unit, lung assessment or coronary care unit) and 21 patients (3.0\%) were transferred to level 3 care (intensive care unit). 74 patients $(10.5 \%)$ were palliated in view of terminal illness.

Similarly, after attending the MET call, 213 patients were given no further cardiac arrest resuscitation, but 94 of these patients were still for MET calls. 54 (60.7\%) patients whose care was escalated were discharged from hospital. Of the total activated MET calls, only 20 patients developed cardiac arrests.

\section{Conclusions}

The MET on-call team made a marked improvement in patient management, through rapid assessment of high-risk patients, timely decision making on ward management, care escalation, and when necessary identifying dying patients and making appropriate plans. The MET on-call team has resulted in a $54 \%$ reduction in the number of cardiac arrest calls; its adoption in all acute hospitals will lead not only to improved patient care, but also to more efficient use of available clinical resources.

\section{Conflict of interest statement}

I certify there are no conflicts of interests with any organisation regarding the material discussed. 